16 | 2013

NOVECENTO... E DINTORNI

« On ne naît pas... on le devient "

\title{
Sotto mentite spoglie. Gender studies in Italia
}

Under disguise. Gender Studies in Italy

\section{Paola Di Cori}

\section{OpenEdition}

\section{Journals}

Edizione digitale

URL: http://journals.openedition.org/cei/1055

DOI: $10.4000 /$ cei. 1055

ISSN: 2260-779X

\section{Editore}

UGA Éditions/Université Grenoble Alpes

\section{Edizione cartacea}

Data di pubblicazione: 30 juin 2013

Paginazione: 15-37

ISBN: 978-2-84310-245-5

ISSN: $1770-9571$

Notizia bibliografica digitale

Paola Di Cori, «Sotto mentite spoglie. Gender studies in Italia», Cahiers d'études italiennes [Online], 16 2013, online dal 15 décembre 2014, consultato il 26 mars 2021. URL: http://journals.openedition.org/ cei/1055 ; DOI: https://doi.org/10.4000/cei.1055 


\title{
SOTTO MENTITE SPOGLIE. GENDER STUDIES IN ITALIA ${ }^{1}$
}

\author{
Paola Di Cori \\ Roma
}

[...] il trickster appare contemporaneamente demiurgo $e$ briccone, astuto $e$ stolto, ingannatore $e$ ingannato [...]. Se c'è per lui una normalità è la sua anomalia, se ha una misura è la mancanza di misura. [...] Nato da parti anomali, insegna agli uomini a partorire; generato da grumi di sangue dà origine alle regolari mestruazioni [...]. La sua natura di essere fuori ordine rende per forza approssimativo e alla fine falsante ogni tentativo di contenerlo entro limiti di una qualsiasi 'normalită di aspetto o di comportamento, d'una qualsiasi regolarità che si definisca per l'esclusione di ciò che la violerebbe.

Silvana Miceli ${ }^{2}$

\section{Un tubero arroventato ${ }^{3}$}

Oltre a quello dell'età non più verde, il privilegio di avviare questo convegno a Grenoble credo possa riferirsi all'impegno con cui ho preso parte fin dalla metà degli anni Settanta alla spericolata impresa di introdurre questi studi in Italia — dai primi passi esitanti fino alla loro buona affermazione nel decennio successivo: una tappa creativa, stimolante e fruttuosa

I. Si tratta della rielaborazione di una relazione presentata al convegno "On ne naît pas... on le devient». I gender studies e il caso italiano, dagli anni Settanta a oggi, tra liberazione sessuale e nuovi tabù. Université Stendhal - Grenoble 3, 25-26 novembre 2010.

2. Silvana Miceli, Il demiurgo trasgressivo. Studio sul trickster, Palermo, Sellerio, 200o, p. 74.

3. Nel corso di queste pagine utilizzo gender, genere, studi delle donne, studi di genere, studi LBGBTQ (lesbici, gay, bisessuali, trans, queer) in corsivo, tra virgolette, in inglese, in italiano, nudi e crudi, per sottolineare la impossibilità - nel contesto italiano, ma non solo naturalmente - di fissare un significato stabilmente convincente da attribuire a queste parole ed espressioni. Cfr. il paragrafo successivo, Gender, genre, genere. 
in particolare dal punto di vista della produzione scientifica e della iniziale configurazione di una inedita identità pubblica per aree della conoscenza che quarant'anni fa erano sconosciute e inesistenti. Non si è trattato di riempire un vuoto: abbiamo invece inventato e costruito qualcosa che prima di allora non esisteva.

$\mathrm{Si}$ è trattato di un lavoro lungo, faticoso e complesso; e se dopo tutto questo tempo è possibile esibire con orgoglio splendidi risultati di ricerca, il bilancio sul piano della trasmissione e della formazione è segnato dalla discontinuità. Da un lato si contano momenti alti di intenso coinvolgimento tra generazioni diverse - nuove riviste e pubblicazioni di qualità, seminari, scuole estive, convegni, dottorati; dall'altro è da lamentare una irregolare presenza e programmazione in molti atenei importanti del paese, e scarse occasioni per le donne giovani di ricoprire ruoli di primo piano ${ }^{4}$.

Volendo rivolgere uno sguardo disincantato alla situazione di fronte a noi, direi che gli studi di genere suscitano oggi pochi entusiasmi e costano tanta fatica; un commento che vale anche per paesi diversi dall'Italia. Trattasi infatti di una condizione condivisa a est e a ovest, nord e sud del mondo, sebbene tradizioni e specificità locali rendano ciascun caso dissimile dall'altro. Nel giudizio scettico si riflette, ahinoi, la crisi profonda dell'intero apparato politico, ideologico e culturale che quarant'anni fa ha fatto da sfondo e sostegno all'affermazione di quei women's studies che più tardi sarebbero diventati gender studies negli Stati Uniti e nel mondo anglofono, per poi da qui servire come base di lancio della loro diffusione nei cinque continenti.

Con qualche venatura malinconica, tutto ciò mi autorizza a una seconda considerazione generale: sono oltre trent'anni, fuori e dentro il Belpaese, che si cerca di fare un bilancio intorno a saperi nati grazie all'entusiasmo profuso da femministe attive nelle università convinte delle immense potenzialità di questi studi di criticare l'idea stessa di sistemazioni ordinate per disciplina e argomenti. Queste risorse energetiche sembrano oggi essere in via di esaurimento, addomesticate e ricondotte a un regime di più tradizionali consuetudini accademiche, tenuto conto dell'aria global-

4. Per una prima rassegna rinvio a Gli studi delle donne in Italia. Una guida critica, a cura di Paola Di Cori, Donatella Barazzetti, Roma, Carocci, 200I. Per quanto la situazione sia mutata rispetto al 2000, quando è stata scritta, si veda la mia introduzione Atena uscita dalla testa di Giove. Insegnare "studi delle donne» e "di genere» in Italia, pp. I5-43; cfr. anche Maria Serena Sapegno, Identità e differenze. Introduzione agli studi delle donne e di genere, Roma, Mondadori Sapienza, 20II. Cfr. infine il fascicolo speciale - a cura di Charlotte Ross e Susanna Scarparo - di «Italian Studies», 65, 2, July 20I0, e il saggio di Ross, Critical Approaches to Gender and Sexuality in Italian Culture and Society, pp. I64-I77. 
mente depressa e deprimente che si respira nella vita politica, culturale ed economica a livello planetario.

Come cercherò di chiarire ulteriormente nel corso di questa relazione, gli studi di genere in Italia si presentano nell'attualità con un marcato profilo di indeterminatezza identitaria. Tra le ragioni di questa fisionomia dai tratti incerti indicherei intanto la tardiva legittimazione istituzionale di questi studi nel paese, avvenuta soltanto dopo il 2000. Un secondo aspetto da tener presente riguarda la disomogeneità di opinioni esistente nel mondo delle donne che lavorano dentro, fuori e intorno all'università. Per molto tempo, pur con motivazioni assai diverse, sia settori influenti del femminismo che esponenti accademiche di riconosciuta autorevolezza hanno manifestato riluttanza più che impegno deciso su modalità e tempi di un inserimento a livello istituzionale di questi studi. La situazione ha cominciato lentamente a sbloccarsi con l'approssimarsi del nuovo millennio, attraverso la riforma universitaria nota come " $3+2 »$, la creazione di molti nuovi insegnamenti, l'ingresso dell'Italia nell'ambito della moneta unica europea e obiettivi comunitari da rispettare anche nell'area della formazione.

Verso la metà degli anni Novanta, nell'area che si richiamava al femminismo il punto di contrasto più acceso ha riguardato il dilemma tra assimilazione, integrazione o autonomia degli studi di genere all'interno dell'organizzazione curricolare. Per oltre quindici anni, dopo ben due tentativi falliti di riformare la 'macchina' didattica, tutte e tre queste opzioni hanno continuato a rimanere questioni irrisolte. Intorno al 1997-98, i conflitti interni (i quali non superavano comunque il livello di modeste scaramucce locali di cui si aveva ogni tanto qualche vaga eco pubblica, trattandosi di un tema ritenuto poco appassionantes) furono presto messi da parte per via di alcuni cambiamenti in corso: da una parte la costituzione di una rete per coordinare le iniziative delle donne europee nel

5. Può servire da esempio un articolo scritto quindici anni fa per promuovere i women's studies e indicare l'opportunità di insegnarli anche in Italia, cfr. Paola Di Cori, Studiando se stesse (titolo redazionale), in «Il Sole 24 ore», 3 marzo 1996. Pochi giorni più tardi fu pubblicata una lunga e polemica risposta di Luisa Muraro - Questione di naso, occhio e orecchio, in "Il Manifesto», 26 marzo I996 - la quale non vedeva quale beneficio sarebbe potuto derivare con la loro introduzione rispetto a quanto le donne stavano già facendo da anni. Intanto, un certo numero di universitarie intraprendenti e meglio inserite nella gerarchia accademica cominciò a prendere parte ai raduni della rete ATHENA. A lungo in Italia si continuò a procedere come su un doppio binario: il rifiuto dell'istituzionalizzazione da un lato in nome della fedeltà alle pratiche femministe degli anni Settanta, e l'obiettivo di essere istituzionalizzate creando una netta separazione da quelle esperienze dall'altro. Cfr. Paola Di Cori, Women's studies. Un caso di identità multipla?, in «Il paese delle donne», I8 febbraio I998. Questa polarizzazione, tuttora esistente, ha avuto effetti negativi perché ha finito per ideologizzare eccessivamente il femminismo e quanto a esso era collegato - testi fondanti, teoria, pratiche orizzontali, tradizioni politiche; e all'altro estremo ha spoliticizzato oltremisura gli studi di genere che nel frattempo si andavano introducendo un po' dappertutto. Si vedano le considerazioni svolte nella seconda parte di questa relazione. 
campo dell'istruzione superiore - ATHENA — alla quale l'Italia prese parte. Il primo appuntamento importante di questo organismo fu la convocazione a Coimbra di una conferenza internazionale nel giugno 1995, riunita per chiamare a raccolta rappresentanti dei vari paesi e valutare insieme nuovi programmi comunitari riguardanti i women's studies; l'incertezza delle posizioni si estendeva anche all'eventuale denominazione da adottare e quindi per tacito accordo si preferiva utilizzare l'inglese che aveva reso questi studi noti in tutto il mondo ${ }^{6}$.

Negli stessi anni la composizione del corpo studentesco negli atenei italiani subiva notevoli modifiche grazie alla presenza di nuove generazioni di donne nate intorno alla metà degli anni Settanta, ormai cresciute sotto l'egida della grande rivoluzione tecnologica e culturale dell'epoca, accompagnata dalla inarrestabile anglicizzazione del linguaggio, dalle accresciute possibilità di viaggiare, di seguire seminari e corsi all'estero e di prendere un dottorato (ricordo che in Italia i programmi di dottorato sono stati introdotti e hanno cominciato a funzionare soltanto dopo il 1984) ${ }^{7}$. Pur da posizioni assai marginali queste giovani, formate e informate sia dentro che fuori d'Italia, finivano anche indirettamente per stimolare cambiamenti di rilievo sul suolo natale nei temi delle ricerche, nelle discussioni pubbliche e negli obiettivi politici; con grandi difficoltà si sforzavano di entrare a far parte della vita universitaria italiana, e intanto cominciavano a organizzarsi in associazioni come quella nota con il nome di "30 something» oltre a partecipare a livello europeo al Next Genderation Network ${ }^{8}$. L'introduzione della riforma del «3+2» alla fine del 1999, grazie anche alla presenza di Laura Balbo al ministero delle Pari Opportunità nel governo allora presieduto da D'Alema, se da un lato ha favorito l'in-

6. Per alcuni eventi e problematiche di quegli anni rinvio alla pubblicazione Donne e Ricerca del CIRSDe (Centro Interdipartimentale di Ricerche e Studi delle Donne) dell'Università di Torino, un bollettino che informava su convegni, didattica e iniziative a livello locale, nazionale e internazionale, uscito regolarmente due volte l'anno dal 1995 al 2000 e che, insieme alla newsletter InOLTRE $i$ corsi costituisce una delle prime espressioni nel paese di coordinamento tra docenti appartenenti a diverse facoltà nella stessa sede universitaria. Cfr. il resoconto della presidente Ursula Isselstein, Coimbra e oltre. Il CIRSDe nel contesto europeo, in "Donne e Ricerca», 2, ottobre I995, pp. 3-IO. Per un resoconto succinto degli appuntamenti di quella che è nota come ATHENA (Advanced Thematic Network for Activities in Women's Studies in Europe) rinvio alla pagina dell'Università della Calabria <www.sociologia.unical.it/women/athena.html> (data di ultima consultazione 8 dicembre 20I2); cfr. inoltre Rosi Braidotti, Parole chiave e problemi nella formazione dei women's studies europei, in Gli studi delle donne in Italia, a cura di Paola Di Cori, Donatella Barazzetti, cit., pp. 79-95.

7. Cfr. Il brutto anatroccolo. Il dottorato di ricerca in Italia fra università, ricerca e mercato del lavoro, a cura di Sveva Avveduto, Sergio Cesaratto et al., Milano, Franco Angeli, 1994, che cerca di tracciare un bilancio a dieci anni dalla sua introduzione.

8. Per le difficoltà che incontrano le giovani femministe italiane rinvio al resoconto di Roberta Corbo, che riassume una situazione diffusa intorno al 1999: Women's studies. Una rete per le giovani universitarie d'Europa, $<$ www.mclink.it/n/dwpress/dwwi62/artı.htm> (data di ultima consultazione 8 dicembre 20I2), nel quale si evidenzia il ruolo propulsivo di Rosi Braidotti all'interno della rete ATHENA con significative ricadute in Italia. 
gresso degli studi di genere nelle università, dall'altro ha coinciso con la crescita dei movimenti no global e un maggiore protagonismo delle giovani, insieme all'emergere di profonde lacerazioni dovute a un confronto mancato tra vecchie e nuove generazioni, aleggiante tra le sparse membra del femminismo come una ferita che non doveva più rimarginarsi.

La rievocazione di un passato prossimo percorso da inquietudini di così diversa natura spiega in parte le difficoltà della situazione attuale, cui occorre aggiungere l'aggravarsi di una emergenza economica servita principalmente per giustificare gli abnormi e intollerabili tagli ai finanzia-

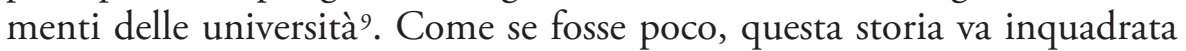
nel contesto di un aberrante lungo inverno pornocratico berlusconiano e dei suoi deleteri effetti sulla rappresentazione e la vita materiale delle donne italiane, della scarsa vivacità del dibattito culturale nel paese e dello stato di crescente degrado dell'università nel suo insieme ${ }^{\mathrm{IO}}$.

Sono quindi consapevole di come, insieme al privilegio, mi sia stata consegnata anche una enorme e ingombrante 'patata bollente', che vorrei tentare di rendere più digeribile con qualche commento anti-agiografico, non limitato soltanto agli importanti traguardi raggiunti — su cui esistono

9. Questo declino non è certo unico dell'Italia, anche se qui ha assunto tratti di patologica gravità per alcuni difetti strutturali ben noti — dal familismo alle forme di cooptazione corporative e corrotte, dalla organizzazione didattica assai arretrata all'invecchiamento del corpo docente. Pessimi sono stati i risultati delle tre riforme che hanno cercato di mettere mano alla disastrata situazione, a partire dalla fine degli anni Novanta con quella del ministro Berlinguer, successivamente corretta nel 2003 dal ministro Moratti, fino a giungere alla contestatissima riforma Gelmini del 20Io. Negli Stati Uniti a metà degli anni Novanta un grande libro di Bill Readings, The University in Ruins, Cambridge (MS), Harvard University Press, 1995, preannunciava l'imminente trasformazione e declino del modello di una università di cultura di stampo tradizionale, sostituita da quello aziendalistico di una università dell'eccellenza. La versione italiana di questa transizione dal culturale all'aziendale ha prodotto un ulteriore abbassamento qualitativo e, in assenza di una priorità per i meritevoli, ha incrementato la selezione sociale; come noto, i giovani di famiglie benestanti da tempo si formano prevalentemente fuori d'Italia. Per uno sguardo più aggiornato sulle conseguenze del passaggio dall'uno all'altro modello universitario nel corso dell'ultimo decennio si veda il giudizio assai critico di un eminente storico dell'età moderna nel recensire una serie di libri recenti sulla situazione negli Stati Uniti, cfr. Anthony Grafton, Our Universities: Why Are They Failing?, in "The New York Review of Books», November 24, 20II. La crisi finanziaria globale ha colpito duramente le università di tutto il mondo, in particolare le aree umanistiche e di scienze sociali, dove sono nati e fioriti gli studi di genere. Rinvio, per valutazioni aggiornate sulla crisi attuale in Nord America, al fascicolo speciale The Humanities and the Crisis of the Public University, "Representations", I, Fall 20II, e in particolare ai contributi di Colleen Lye, Christopher Newfield, James Vernon, Humanists and the Public University, pp. I-I8, e al saggio di Wendy Brown, The End of Educated Democracy, pp. I9-4I. Sulla pornocrazia berlusconiana rinvio a Paola Di Cori, 2009-20II: un biennio pornocratico. Intorno a Pornotopia di Beatriz Preciado, in Paola Di Cori, Asincronie del femminismo, Pisa, ETS, 2012, pp. 219-239, e Paola Di Cori, Segreti e bugie. Intorno alla Pornotopia di Beatriz Preciado, in «DWF», 2, aprile-giugno 20II, pp. 43-53.

Io. Sulle croniche disfunzioni delle università italiane rinvio alle due raccolte Chi governa l'università? Il mondo accademico italiano tra conservazione e mutamento, a cura di Roberto Moscati, Napoli, Liguori, 1997, e Come e perché cambiano le università in Italia e in Europa: nuove politiche dell'istruzione superiore e resistenze al cambiamento, a cura di Roberto Moscati, Napoli, Liguori, 20Io. Innumerevoli gli interventi di giornalisti e docenti intorno ai concorsi e alle pratiche di cooptazione nepotistica; tra le inchieste più recenti cfr. Paolo Casicci e Alberto Fiorillo, Scurriculum. Viaggio nell'Italia della demeritocrazia, Reggio Emilia, Aliberti, 201 . 
ormai libri e ricognizioni accurate - ma esteso ad alcuni problemi che nelle occasioni di bilancio generale rimangono di solito in secondo piano.

\section{Gender, genre, genere}

Il calore incandescente intorno ai temi che qui ci stanno a cuore comincia ad avvertirsi fin dalla parola «genere»; un termine, come noto, assai complicato da spiegare e da tradurre, le cui caratteristiche, diverse per ciascun contesto linguistico-geografico, bisognerebbe specificare di volta in volta. Nel programma di questo convegno, per esempio, si usano tre lingue diverse come se fossero una sola, all'interno di una gerarchia dove l'inglese è all'apice, e in affannata rincorsa italiano e francese si qualificano a pari merito, visto che siamo a Grenoble e ci occupiamo di cose italiane; e tutto ciò come se le parole di ogni lingua fossero traducibili nelle altre due senza fatica o specificazioni, con uno sforzo minimo volto a cercare assonanze fonetiche piuttosto che significati equivalenti.

Personalmente, ho sempre insistito sull'importanza del carattere difficilmente definibile di studi che prima erano «delle», «sulle», donne; e poi sono diventati «di genere». Per non parlare del più recente 'ombrello' lessicale racchiuso nel termine queer. Occorre infatti ricordare che i tanti disaccordi e contrapposizioni intorno al significato più 'esatto' e 'corretto' delle parole quando sono tradotte da una lingua all'altra — su come rendere «gender» si discute appassionatamente in tutto il mondo fin dalla conferenza mondiale delle donne di Pechino del 1995 - riflettono qualcosa a suo tempo sottolineato da Roland Barthes nelle lezioni sulla retorica antica. In quel testo dei primi anni Settanta il grande studioso scriveva che «il linguaggio è un potere», e proprio per questo «si sono dettate regole selettive d'accesso a questo potere, costituendolo in pseudoscienza, chiusa a "quelli che non sanno parlare"». Nell'antichità — aveva affermato Barthes — «l'arte della parola è originariamente legata ad una rivendicazione di proprietà» ${ }^{\mathrm{II}}$.

Come suggeriscono numerose pubblicazioni recenti, questo desiderio di affermazione proprietaria si ripresenta con forza nel presente, seppure esibendo il suo versante opaco di grande inquietudine intorno a dove e chi detiene qualche forma di potere; le instabilità di ogni tipo e il clima di

II. Roland Barthes, La retorica antica, Milano, Bompiani, 20II (edizione originale 1970). Si tratta di un seminario tenuto nel 1964-1965 presso l'Ecole Pratique des Hautes Etudes; le citazioni sono rispettivamente alle pp. 8 e I4. 
incertezze che caratterizzano le società in cui viviamo si riflettono inevitabilmente sul modo in cui parliamo. Negli ultimi decenni, infatti, la problematica natura della sovranità e dell'autorità si rispecchia nelle parole in uso, che appaiono imprecise e sguarnite. Mentre assistiamo inermi al loro affannarsi per offrire definizioni rassicuranti e dall'apparenza stabile intorno a ciò che dovrebbero rappresentare, il linguaggio sembra aver perso ogni pretesa di costituire un solido terreno su cui porre dei fondamenti: attraversarlo è come camminare sulle sabbie mobili ${ }^{\mathrm{I2}}$. Proprio questo è da tenere in mente quando parliamo degli studi di genere.

$\mathrm{Al}$ di là delle vicende che hanno accompagnato la diffusione e l'uso del termine «gender», e al fatto che spesso contemporaneamente alterniamo questa parola con altre, in sostituzione o come sinonimi, a riprova di una continua instabilità e ambivalenza nell'uso corrente, bisogna comunque tener conto del fatto che fino a oggi non abbiamo ancora trovato un termine altrettanto flessibile e polivalente per indicare soggettività umane sessuate mutevoli nel tempo. Da una parte, come aveva già scritto a suo tempo Joan Scott, «gender» è parola dall'aria innocua, ha un aspetto compassato e ammodo; viene accettata dai benpensanti e dai consigli di facoltà, da ministeri, assessorati e giunte comunali, che mai vorrebbero sporcarsi con iniziative o programmi riguardanti 'sessi' e 'sessualità', anche se ormai in molte scuole si insegna educazione sessuale, e tenuto conto del fatto che fino al novembre $201 \mathrm{I}$ abbiamo vissuto sotto un regime pornocratico durato ben diciassette anni — del quale tutto si può dire, tranne che si sia distinto per le sottigliezze nell'uso del linguaggio. Dall'altro lato, una delle prerogative da valorizzare in questa parola è proprio l'indubbio carattere plastico e oscillante nel tempo e nello spazio che essa ostenta con tanta disinvoltura ${ }^{13}$.

I2. Si veda per esempio Gustavo Zagrebelsky, Sulla lingua del tempo presente, Torino, Einaudi, 20Io, in cui l'autore osserva che per quanto non viviamo all'interno di un universo linguistico governato totalitariamente, come quello descritto nel mirabile diario scritto durante il nazismo dal filologo Klemperer sulla lingua del Terzo Reich, "siamo immersi in una lingua che ci sovrasta, elaborata e diffusa nei circuiti della comunicazione, carica di sottintesi che ci avvolgono come in un intreccio di significati che sembrano indipendenti da noi, perché li accogliamo come ovvi, non contestabili, e dotati di una propria persuasività che non ha bisogno di essere dimostrata ogni volta» (p. 9). Cfr. inoltre Victor Klemperer LTI. La lingua del Terzo Reich. Taccuino di un filologo, Firenze, Giuntina, 1999 (edizione originale 1947), e Raffaella De Santis, Le parole disabitate. Il Novecento, Torino, Aragno, 2orI, la quale sostiene tuttavia la tesi opposta, e invece di considerare il linguaggio al centro di un più complesso insieme di fenomeni legati anche ad alcune profonde modificazioni della vita pubblica, pensa alle parole come case svuotate e non come spazi sovrappopolati, attraversate da molteplici intenzioni, sulle quali si esercitano poteri di esclusione, discriminazione, deformazione — mai così evidenti come negli ultimi anni del governo di Berlusconi.

13. Rinvio per ulteriori chiarimenti alle pagine su questi temi in Paola Di Cori, Asincronie del femminismo, cit. 
La natura malleabile, soggetta a continue modificazioni dei termini «genere/gender/genre» nelle tre lingue si manifesta nella loro disposizione a collocarsi a metà strada tra sostantivo e aggettivo; essi sono utilizzati sia per definire che per descrivere. Indicano un fattore relativo alle identità sessuali, e anche l'intenzione di rimetterle in discussione insinuando elementi critici. Suggeriscono il tentativo di delucidazione preliminare, un inizio; e allo stesso tempo mostrano anche il problema su cui interrogarsi, un'inchiesta da avviare. La parola "gender» ha un inesauribile bisogno di essere rispiegata, risignificata, decostruita e ricostruita incessantemente. $\grave{E}$ questa la sua croce e delizia, virtù e tormento per tutte/i noi.

Non a caso, nelle prefazioni e commenti ex-post a ogni nuova discussione e ristampa dei loro testi fondatori - alcuni assai recenti — Joan Scott e Judith Butler (sarebbe importante che francesi e italiane facessero la loro parte per i rispettivi contesti socio-linguistici) sottolineano spesso il dato di inesauribile problematicità con cui, fin dalla seconda metà degli anni Ottanta, entrambe avevano voluto caratterizzare la parola e i saperi che da essa sono derivati ${ }^{14}$. Un elemento che non ha impedito lo straordinario sviluppo di questi studi nel mondo anglofono e fuori di esso; anche se, come siamo qui pienamente consapevoli, ciò ha significato in molti casi una istituzionalizzazione e indebolimento della sua originaria fisionomia trasgressiva. Quello che importa è continuare ad alimentare gli usi critici di «gender», visto che a partire dagli anni Novanta — ha ancora insistito Joan Scott in un recente fascicolo di «Diogène» — almeno negli Stati Uniti, la parola «non ha più creato sconcerto, non si è più presentata come una sfida al linguaggio grammaticale stabilito» ${ }^{15}$.

Ma quel che vale per l'inglese negli Stati Uniti, non necessariamente è equivalente a quanto accade negli altri contesti che qui ci riguardano più da vicino. L'irruzione di termini provenienti da altre lingue in quella 'nazionale' parlata abitualmente è un'operazione in cui eccelle, da alcuni decenni a questa parte, l'invasivo e invadente inglese; e di cui, caso per

I4. Il saggio di Joan Scott — Gender: A Useful Category of Historical Analysis — è stato pubblicato per la prima volta sulla "American Historical Review», 9I, 5, December 1986, pp. I053-I075; cfr. il recente dibattito a più voci intorno a questo saggio riguardante gli esiti e le potenzialità non ancora espresse: AHR Forum: Unanswered Questions, in "American Historical Review», II3, 5, December 2008, pp. I422-I430 e la recente raccolta a cura di Judith Butler e Elizabeth Weed, The Question of Gender. Joan W. Scott's Critical Feminism, Bloomington (IN), Indiana University Press, 20II. Scott ha aggiunto una prefazione alla edizione riveduta del 1999. Gender Trouble di Judith Butler è stato pubblicato nel I990, cfr. la seconda edizione, London, Routledge, I999, e le pagine che precedono quest'ultima, pp. viı-XXvi.

I5. Joan Scott, Le genre: une catégorie d'analyse toujours utile?, in «Diogène», 225, janvier-mars 2009, pp. 5-I4; 9 (traduzione mia). 
caso, occorrerebbe approfondire vantaggi e rischi ${ }^{16}$. In Francia, la commissione che controlla la terminologia e i neologismi, sulla base della sua estraneità ai criteri nazionali che presiedono l'uso corrente della lingua, ha stabilito nel 2005 che gender non è una parola francese, a dispetto dell'uso crescente che ne viene fatto. Ma in barba a cotanta sicurezza, pochi anni dopo, nel 2008, veniva pubblicato (da un editore belga con succursale a Parigi) un manuale di Introduction aux Gender Studies che ignorava l'autorevole avvertimento e spazzava via ogni residuale incertezza in proposito; anche in Francia si sono quindi dovuti rassegnare ${ }^{17}$. A meno di voler reintrodurre metodi tipici dei regimi totalitari, non si può certo impedire ai parlanti di esercitare il proprio diritto a servirsi dei termini che ciascuno sceglie come i più indicati per esprimersi ${ }^{18}$.

Su un altro versante, sollevare problemi riguardanti eventuali imprecisioni e la difficile resa di "gender» in lingue diverse dall'inglese non è un'impuntatura né un richiamo a usare soltanto le parole della propria lingua nazionale. Si rivela invece assai utile per sottolineare ancora una volta l'assoluta supremazia statunitense in quest'area di studi; e anche per mettere in risalto la centralità della traduzione con il suo seguito di problemi annessi e connessi. In un certo senso, essere consapevoli dell'esistenza di un margine di incertezza ogni volta che la parola viene utilizzata consente di mantenere aperto uno spazio di potenziale agentività ${ }^{19}$ da parte

I6. Elias Canetti non ha esitato a riferirsi al carattere passionale delle parole. In alcune pagine del i969 sulla condizione di chi emigra in un altro paese, l'Inghilterra nel suo caso, e continua a scrivere e parlare in tedesco, ha ricordato il tormentato irrompere di parole della lingua d'origine in quella di accoglienza, e il turbamento provocato da queste intrusioni dell'una dentro, contro, insieme all'altra: «Da allora — scrive — non posso più dubitare che le parole siano dotate di una loro speciale carica passionale. In realtà sono come le persone, non tollerano di essere trascurate o dimenticate. A prescindere da come sono custodite, rimangono in vita e saltan su all'improvviso per reclamare i loro diritti.» Cfr. Elias Canetti, Accessi di parole, in La coscienza delle parole, Milano, Adelphi, I984 (edizione originale 1974), pp. 229-237; 234. Judith Butler, a sua volta, si è concentrata sul potere offensivo delle parole analizzando situazioni in cui i soggetti sono sottoposti a una particolare «vulnerabilità linguistica» — basti pensare alla quotidiana violenza esercitata dagli insulti razzisti, omofobi, misogini, antisemiti contro donne e uomini appartenenti a gruppi e minoranze discriminati, immigrati, anziani e malati. Cita il discorso di accettazione del premio Nobel per la letteratura pronunciato da Toni Morrison nel I993, quando afferma: «Il linguaggio oppressivo non si limita a rappresentare la violenza: è violenza», in Judith Butler, Parole che provocano. Per una politica del performativo, Milano, Raffaello Cortina, 2010 (edizione originale I997), p. 8.

17. Introduction aux Gender Studies: Manuel des études sur le genre, a cura di Alexandre Jaunait, Laure Bereni et al., Bruxelles, De Boeck, 2008.

I8. Oltre al lavoro di Klemperer sulla Germania nazista ricordato precedentemente, e le considerazioni di Canetti, si veda la splendida ricerca di una linguista e critica letteraria nordamericana sulle parole proibite e quelle consentite durante la dittatura militare argentina negli anni 1976-1983. Cfr. Marguerite Feitlowitz, $A$ Lexicon of Terror: Argentina and the Legacies of Torture, Oxford, Oxford University Press, I998.

19. Questo è il termine scelto per rendere in italiano il tormentato agency dalla studiosa che ha compilato la voce corrispondente inclusa in un ottimo dizionario di etnolinguistica. Cfr. Laura M. Ahearn, Agentivitàl Agency, in Culture e discorso. Un lessico per le scienze umane, a cura di Alessandro Duranti, Roma, Meltemi, 200I, pp. I8-23. 
di coloro che la utilizzano come fosse 'merce d'importazione'. Conta in modo speciale adoperarla persuase/i delle sue ambivalenze e consapevoli della potenziale forza critica che sprigionano le parole; e anche dell'importanza di tener conto delle "comunità di pratiche» che esercitano pressioni su come si usa il linguaggio ${ }^{20}$. Basta pensare agli equivoci derivati dalla errata titolazione in italiano di testi teorici fondamentali come quelli di Judith Butler o di Eve Kosofsky Sedgwick, per citare esempi clamorosi, fuorvianti fino alla deformazione e al rovesciamento del significato originario $^{21}$.

Se alcune strade sono in apparenza sgombre da impedimenti linguistico-burocratici, parlare di "gender studies» rimane in ogni modo una questione non del tutto ovvia e dai significati tutt'altro che condivisi e inequivocabili. Era così anche trenta e quarant'anni fa, quando si parlava di «studi delle donne» $\mathrm{e}$ in Italia erano in poche a sapere di cosa si trattava.

Pensiamo a quel che accade in tempi più vicini con $\mathrm{i}$ «queer studies». Per sottolineare le molte potenzialità ancora da esplorare di un'area cresciuta a dismisura fin dai primi anni Novanta, la rivista "Social Text» intitolava nel 2005 un fascicolo doppio: What's Queer about Queer Studies Now? La domanda coglieva nel segno negli Stati Uniti, anche se aveva poco senso in Italia, dove fino a poco tempo fa erano assai poche/i a sapere qualcosa di «queer studies», in buona parte provenienti da studi intorno alle culture in lingua inglese. L'erosione dei margini di incertezza e trasgressione insiti nella fase iniziale di introduzione di alcuni termini veniva bene evidenziata dal saggio introduttivo di Eng, Halberstam e Muñoz, che prendeva atto di come le valenze provocatorie di parole e categorie erano destinate a perdersi una volta che si istituzionalizzavano all'interno di specifiche aree di studio ${ }^{22}$. Accanto e insieme ai molteplici significati

20. Cfr. la voce Genere/ Gender scritta da Mary Bucholtz, in Culture e discorso. Un lessico per le scienze umane, a cura di Alessandro Duranti, cit., pp. I22-I25, la quale suggerisce l'utilità di fare attenzione al modello linguistico delle "comunità di pratiche», «format $[\mathrm{e}] \mathrm{da}$ individui temporaneamente uniti in virtù del loro comune impegno in una particolare attività, che in tal modo sono in grado di cambiare identità ad ogni istante. Questo tipo di approccio tende a far decrescere l'importanza del genere come categoria esplicativa primaria (al pari di ogni altra dimensione del sé), promuovendo l'immagine di identità fluide, situate e fondate sulle attività» (p. I25). Cfr. anche Reinventing Identities: The Gendered Self in Discourse, a cura di Mary Bucholtz, A. C. Liang, Laurel A. Sutton, New York, Oxford University Press, 1999.

2I. Mi riferisco alle imprudenti traduzioni di Gender Trouble (1990) e Undoing Gender (2004) di Butler, tradotti rispettivamente come Scambi di genere (Firenze, Sansoni, 2004) e La disfatta del genere (Roma, Meltemi, 2006), e alla recente edizione di Epistemology of the Closet di Sedgwick, che si è trasformato nell'incomprensibile Stanze private (Roma, Carocci, 20II).

22. Sulla inevitabile tensione tra iniziali impulsi rivoluzionari e successivi assestamenti riformatori, tipica di tutti i grandi movimenti sociali e culturali, si soffermano numerose considerazioni di bilancio generale sugli studi di genere. Cfr. i contributi raccolti in Women's Studies on the Edge, a cura di Joan Scott, Durham (NC), Duke University Press, 2008. 
attribuiti a vocaboli italiani o stranieri da parte di chi li adopera, alla base delle continue modifiche del loro raggio di significazione, si aggiunge un fenomeno di invecchiamento; con il passare del tempo l'uso continuo logora qualsiasi intenzione eversiva iniziale e smussa ogni asperità neutralizzando le parole, le quali acquistano progressivamente un'aria di naturale assestamento dentro il linguaggio comune.

\section{Spaesamenti}

D'altra parte, a fronte di condizioni e difficoltà comuni a tutte e tutti quelli che utilizzano questi termini - in inglese e in altre lingue — vale la pena di sottolineare alcune specificità locali. Tra le più evidenti spicca un immediato dato di spaesamento e di incertezza: in Italia la fisionomia degli studi di genere è tutt'altro che ben definita. Una ricognizione nelle pagine web di molti atenei italiani sparsi lungo il paese conferma il fatto che essi sono diffusi un po' ovunque, e nella proliferazione dell'offerta didattica verificatasi nelle università dopo il 2000 vengono ormai accettati da un establishment accademico poco reattivo, che li accoglie a seconda dei casi con rassegnato paternalismo e/o indifferenza. Al tempo stesso, molte volte si ha l'impressione di una perenne oscillazione tra l'endemica assenza di efficaci moduli introduttivi su un versante, e sull'altro il gran numero di corsi monografici e di insegnamenti esistenti a livello specialistico. Spesso i programmi dei libri da portare all'esame sono a dir poco scarni e inconsistenti; è facile capire che la/lo studente in molti casi si limiterà a leggere un centinaio di pagine o poco più di carattere illustrativo generale; oppure annasperà disorientata/o all'interno di una monografia specialistica sui temi più svariati firmata dal/dalla docente. A contrapporsi a questa diffusa genericità esistono da anni corsi, seminari, master e cicli di dottorato interuniversitari di ottimo livello, come attestano i buoni risultati di ricerca raggiunti nell'ultimo ventennio da molte e molti giovani che si sono formati all'interno di questi programmi; segno evidente che nel periodo più recente sono stati fatti grandi sforzi per costruire percorsi di studio assai seri, che poco hanno da invidiare a quelli di altri paesi ${ }^{23}$.

23. Così attestano da anni i seminari e iniziative promossi dai maggiori centri interdipartimentali esistenti — a Torino, Trento, Padova, Bologna, Milano, Roma, Napoli, Palermo, e in molte altre sedi — come anche gli appuntamenti periodici, i convegni e le pubblicazioni sotto l'egida delle due principali associazioni di area umanistica - la Società delle Storiche (dal 1989) e la Società delle Letterate (dal I995). 
In molti casi, tuttavia, è difficile farsi un'idea precisa di cosa venga insegnato, come e da quale tipo di docenti. Gran parte di questi insegnamenti ha dei programmi assai vaghi: i riferimenti al femminismo, la sensibilità e conoscenza delle problematiche che circondano gli studi LGBQT possono essere molto evidenti, oppure inesistenti; i testi adottati variare da un minimo composto da compilazioni e rassegne affrettate, a un massimo costituito da monografie scientifiche raffinate ma eccessivamente complesse, del tutto inadeguate a funzionare da introduzione. Talvolta i e le docenti nel proprio percorso di lavoro si sono occupati di altro, ma nella più recente pubblicazione, per dare mostra di apertura al progresso, hanno aggiunto un breve capitolo riguardante «le donne» $\mathrm{o}$ «le identità sessuali»; possono così assegnare il libro in questione come testo d'esame e il loro corso finirà compreso tra quelli relativi agli studi di genere.

Nelle università italiane coesistono e confliggono alternativamente iniziative di alto profilo e corsi assai modesti; e così ottimi programmi di ricerca e di formazione superiore, efficaci insegnamenti introduttivi su specifici aspetti di un universo conoscitivo ormai sterminato esistono accanto e insieme ai prodotti di una offerta didattica generica, frammentata, indefinita, spesso del tutto insufficiente a garantire una buona strumentazione di base, entro la quale insegnamenti di argomento affine sono spesso scollegati l'uno dall'altro e privi di un indispensabile momento di raccordo generale; in alcuni casi sono soprattutto i periodi di studio all'estero a offrire un rimedio alle croniche défaillances della formazione in Italia. Così descriverei in prima approssimazione $\mathrm{i}$ «gender studies» nel Belpaese.

Come si può in parte comprendere da tutto ciò, è poco utile parlare degli studi di genere in Italia senza fare riferimento alla struttura e funzionamento dell'università italiana nel suo insieme, oggetto negli ultimi anni di innumerevoli critiche culminanti nell'infausto progetto di riforma che porta il nome del ministro Gelmini, considerato da buona parte del corpo accademico e studentesco come una vera e propria sentenza di morte. Non si tratta solo dei ben noti e gravissimi problemi di familismo, conformismo, invecchiamento del corpo docente, corruzione, mancanza di fondi, ecc., né dell'applicazione di criteri di valutazione del tutto opinabili e imprecisi, in particolare per l'area umanistica. Questo è solo il lato più visibile. Un fattore rilevante di cui raramente si parla riguarda piuttosto le pratiche didattiche, i contesti dell'apprendimento e della trasmissione culturale. Per dirla in altre parole, le tradizioni di insegnamento e formazione delle università italiane, iniziato ormai da oltre dieci anni il nuovo millennio, mostrano un volto solo fintamente rinnovato. Conservano infatti 
un ottocentesco spirito dominato dal soffocante ossequio alle gerarchie e dai vincoli inestirpabili con i modelli didattici antiquati delle lezioni frontali: in essi la comunicazione tra le generazioni, tra studenti e docenti, e di questi ultimi tra di loro intorno alle proprie pratiche è scarsa; la partecipazione attiva del corpo studentesco nel suo insieme assai debole, modesto e quasi nullo l'impegno di lettura e scrittura per chi frequenta, accanto alla possibilità di non frequentare; assente un riscontro efficace sulla preparazione e capacità comunicativa dei/delle docenti; le prove d'esame restano prevalentemente orali; le tesi e tesine di laurea spesso sono il frutto di compilazioni affrettate messe insieme con brani strappati alle pagine del web; e molto altro ancora.

Questo sfondo va tenuto presente nel caso di aree di ricerca a forte caratterizzazione politica come sono gli studi delle donne e di genere, i quali possono facilmente trasformarsi in veicolo di ideologia e diventare facile bersaglio da parte di chi - donne o uomini, femministe, gay, lesbiche o no, qui pari sono - non ha alcun interesse o desiderio personale di promuoverli. Su un versante opposto, come accade in molti corsi di scienze sociali, essi vengono neutralizzati. Nell'affanno per allontanare l'ombra minacciosa dell'ideologia e della propaganda politica, si sterilizzano e raffreddano argomenti incandescenti di cui gli studi queer e di genere sono pervasi, e intorno ai quali hanno costruito la propria ragion d'essere.

Da ciò deriva una constatazione non banale riguardante la natura tutt'altro che innocua degli studi che ci stanno a cuore: non è possibile fare finta che ci occupiamo di un argomento neutro, che parlare di «gender, gay, lesbian» e «queer studies» sia come parlare di numismatica, di archeologia medievale o di fisica delle particelle elementari. Come scrive Derrida, "fingendo di considerare naturale ciò che non lo è e non lo è mai stato, si neutralizza. Si neutralizza cosa? Piuttosto si dissimula, in effetto di neutralità, l'intervento attivo di una forza e di un apparato. [...] Non c'è un posto neutro o naturale nell'insegnamento» ${ }^{24}$.

Inoltre, quando si dice «studi LGBTQ», in tutto il mondo si intende come fosse cosa risaputa che si tratta di insegnamenti impartiti prevalentemente nelle università. Ma questo dato non è così ovvio per l'Italia, un paese dove le istituzioni accademiche da sempre - nelle facoltà umanistiche — raramente hanno offerto buone occasioni per l'introduzione di

24. Jacques Derrida, Dove comincia e come finisce un corpo insegnante, in François Châtelet, Jacques Derrida et al., Politiche della filosofia, a cura di Dominique Grisoni, Palermo, Sellerio, 2003 (edizione originale 1979), pp. 40-70; 42 . 
tematiche transdisciplinari e per confronti orizzontali. È noto infatti che le sedi più adatte per l'aggiornamento e l'informazione colta intorno alle pratiche sociali si trovano spesso all'esterno delle università: nelle centinaia di centri culturali, nei vari festival di letteratura, audiovisivi, cinema, storia, filosofia, arti, matematica e scienze che si tengono nelle piazze e negli auditorium distribuiti sia a nord che a sud, o in alcuni programmi come quelli trasmessi da RadioTre, seguiti con partecipazione spesso entusiasta da un pubblico di migliaia di persone mediamente assai qualificato; che gran parte dei temi più stimolanti di dibattito sono sollevati da un elevato numero di donne e uomini estranei al mondo accademico, nelle pubblicazioni online o di nicchia, pagine Facebook e nello spazio infinito delle reti informatiche. Ormai da anni in Italia si ha la sensazione che dentro le università si impari poco: è fuori, all'esterno e all'estero, che si respira un'aria meno sussiegosa e ammuffita. Non è casuale che siano proprio gli studi di cui stiamo parlando ad avere evidenziato il ruolo basilare e l'apporto cruciale delle pratiche sociali, sessuali, politiche nel rinegoziare aggiornamenti e nuovi significati da attribuire agli oggetti che studiamo ${ }^{25}$.

Per molto tempo, dai primi anni Ottanta in avanti, coloro che hanno promosso l'avvio e l'affermazione di studi delle donne e di genere hanno vissuto lacerate/i da un interno tormento; da una parte erano, e sono, animate/i dal profondo desiderio di introdurre tematiche e concettualizzazioni provenienti non più e non solo dalle militanze, ma in buona misura dall'effervescente produzione intellettuale di lingua inglese, in misura inferiore francese e ormai internazionale; dall'altro lato, oggi ancor più di ieri si trovano a essere fortemente condizionate/i dalla necessità di ottenere una legittimazione all'interno del corpus accademico e di riuscire ad avanzare nella carriera, almeno quel tanto necessario a non essere totalmente schiacciate/i ed emarginate/i da una istituzione ostile al cambiamento, la quale subisce le ondate burocratico-riformatrici con scarso spirito reattivo. Questo duplice vincolo caratterizza gli studi di genere un po' in tutto il mondo ${ }^{26}$, ma le vicende assai prolungate di se e come introdurli offrono alcune chiavi di interpretazione interessanti per il caso italiano, e consentono di comprendere qualcosa di più di quanto accaduto e accade a quanti si ostinano a praticarli nel Belpaese.

25. Sul rapporto tra dentro e fuori si sofferma la bella introduzione di Charlotte Ross e Susanna Scarparo al fascicolo di «Italian Studies», cit., pp. I60-I63, che ne vedono soprattutto i risvolti positivi, mentre io qui cerco di sottolinearne alcuni aspetti meno visibili.

26. Si vedano le osservazioni in proposito di Joan Scott, Introduction: Feminism's Critical Edge, in Women's Studies on the Edge, a cura di Joan Scott, cit., pp. I-I3. 


\section{Invenzione e travestimento}

Non ci sono dubbi, l'argomento di questo convegno si presenta come un oggetto in parte misterioso. Se in altri continenti e aree geografiche anche vicine la storia di come gli studi di genere sono nati, si sono sviluppati, diffusi, introdotti, insegnati, sembra relativamente facile da delineare, in Italia lo è molto meno. Come siamo riuscite/i a trasmettere quell'immenso patrimonio di impegno politico e di pratiche sociali che sono stati il femminismo e i movimenti gay, lesbico, queer, trans, alle altre generazioni? Come rinnoviamo questo patrimonio e queste esperienze con nuovi contenuti? Quali possono essere gli obiettivi nuovi da porsi nel clima odierno caratterizzato dall'incertezza economica e sociale, dai drammi politici, dal disordine istituzionale, dal conformismo e dalla confusione intellettuale, dal gigantesco frullatore mediatico che tutto mescola e sbriciola, dove soltanto lavorare ai margini, o nel sottosuolo, sembra dare qualche temporaneo sollievo?

È utile a questo punto aprire una breve parentesi per ricordare come si lavorava soltanto trent'anni fa. Prima della rivoluzione tecnologica, di internet e dei viaggi low cost, occorreva affrontare alcuni ostacoli pratici di cui oggi si è quasi persa la memoria: usavamo ancora la macchina da scrivere, telefonare all'estero o in altre città era molto caro e quindi si ricorreva alle lettere e alla posta, si viaggiava assai di meno, con voli charter $\mathrm{o}$ in seconda classe su treni scalcagnati. Le riviste in inglese più importanti come «Signs», «Feminist Studies», «Feminist Review» erano difficili da trovare in Italia, così i libri; ce li scambiavamo come reliquie, e intanto nelle redazioni di «DWF» e «Memoria» si cercava di selezionare e tradurre gli articoli che sembravano più importanti. A ripensarci adesso - mentre digito sul computer, stampo tre pagine in pochi secondi, apro l'e-mail e consulto Wikipedia - era un lavoro di scavo e di ritrovamenti fatto con scarsissimi mezzi materiali, tempi imprevedibili e spesso molto lunghi; senza contare che le biblioteche italiane di allora erano quasi inservibili per coloro che decidevano di occuparsi di studi di genere (e anche di altro). Chi se lo poteva permettere cercava di andare a studiare a Londra o negli Stati Uniti, ma negli anni Ottanta erano ancora in poche a farlo.

Volendo ricostruire l'atmosfera di quei tempi neanche troppo lontani, i ritardi, le lentezze, le disponibilità materiali limitate sono elementi di grande importanza per tracciare un bilancio su ciò che si è fatto. La controparte positiva di tutti questi ostacoli era la convinzione di stare inventando qualcosa di cui non si sapeva quasi niente, non si capiva bene cosa fosse, dove collocarla e come svilupparla. $\grave{E}$ un dato all'origine di una sensazione allora diffusa, difficile da definire, che descriverei sinteticamente come una 
miscela di entusiasmo e di immaginazione, la combinazione indispensabile per inventare dal nulla; una unione che ha segnato positivamente e in profondità la mia generazione. Abbiamo lavorato infatti per alcuni anni muovendoci a tentoni, prive di una autorità cui fare riferimento, insinuandoci nelle pieghe dei pregiudizi dei colleghi e delle riluttanze di altre donne, dentro strutture malferme, invecchiate, opprimenti; ma l'ambizione di riuscire a costruire qualcosa nel deserto era una sfida irresistibile. Raggiungere questo obiettivo rimane il principale risultato ottenuto dalle femministe attive nell'università nel periodo aurorale degli studi di genere.

Se l'orgoglio di questa rivendicazione è comune a donne di molti paesi, ci sono alcune differenze di rilievo del caso italiano che occorre sottolineare. Almeno per i decenni '70-'9o, oltre i confini del paese i "women's» e i "gender studies» si sono affermati e sviluppati «fuori dall'armadio», assumendo denominazioni varie in maniera aperta: «studi delle donne», «storia delle donne», «studi femministi», «studi di genere», «studi gay e lesbici» e così per tutte le diverse denominazioni via via adottate. Le donne che li hanno avviati e promossi sono state anche le principali sostenitrici a livello istituzionale di questi studi; non solo con le loro ricerche ma soprattutto con il loro impegno a livello di politica accademica. Inoltre, nel caso statunitense e francese il primo piano era occupato da alcune figure assai rispettate scientificamente, le quali si prendevano il compito e la responsabilità degli estenuanti ma indispensabili confronti, trattative, conflitti, compromessi - con le istituzioni, con le amiche, con le e i colleghi più giovani e meno affermati.

In terra italica la questione si è rovesciata. Per decenni si sono tenuti corsi e seminari di studi delle donne e/o di genere, anche affollatissimi; ma si trattava sempre di iniziative che vivevano in una speciale condizione di semi-clandestinità apertamente consentita, poiché tutti — dal bidello al rettore - erano al corrente di cosa si trattava. Si insegnavano nascosti sotto una impersonale etichetta legittima, dietro la quale si poteva trovare un affascinante corso su Luce Irigaray, o sul lavoro delle donne nel '700, la circolazione di beni nelle società matrilineari delle Trobriand, la segregazione delle donne nel mercato del lavoro, Ildegarda di Bingen, Virginia Woolf e Olympe de Gouges, Joan Robinson e Marie Curie, ecc. Ciascuna docente si muoveva impacciata, costretta a negoziare faticosamente nei consigli di facoltà (per chi aveva il privilegio di farne parte; ed erano pochissime fino a tutti gli anni Novanta); per non parlare di una presenza femminile nei senati accademici, vent'anni fa quasi inesistente ${ }^{27}$.

27. Si vedano per quel periodo i dati pubblicati dall'ISTAT, Donne all'università, Bologna, Il Mulino, 20oI; dieci anni dopo, un articolo di Rita Biancheri, Carriere femminili e università, del I3 dicembre $2010-<$ www. 
Fino al 2000 le studiose femministe hanno fatto ricerca e insegnato 'sotto mentite spoglie', prive di nome, impossibilitate a esibire la propria carta d'identità. Gli studi delle donne li abbiamo dovuti non solo inventare, ma anche continuare a reinventare un anno dopo l'altro, come se ogni volta fosse stato necessario presentare di nuovo i documenti per dimostrare la validità di nome, data di nascita e domicilio di residenza.

Prima che si chiamassero «di genere», per indicare molte altre cose, oppure anche soltanto essere utilizzati come sinonimo dall'apparenza 'perbene', gli studi delle donne li abbiamo insegnati en travesti. Ciascuna di noi agiva come un agente segreto mascherato dentro, dietro e sotto un'altra denominazione ufficiale; la quale poteva essere: letteratura italiana, economia politica, antropologia, sociologia, storia moderna, filosofia medievale o storia della scienza. Il documento d'identità legalmente autorizzato per la pratica didattica serviva a coprire i riferimenti a oscuri e disdicevoli commerci sessuali e politici che avrebbero fatto un'oscena irruzione se si fosse usato il loro vero nome.

\section{Dal basso}

Inoltre, va anche ricordata un'altra differenza con quanto accaduto all'estero, motivata sempre dalla questione del travestimento. Gli studi delle donne, femministi, e poi di genere, lesbici, gay e queer sono nati e cresciuti dal basso; con alcune eccezioni il più delle volte erano promossi da ricercatrici e studiose precarie. Fino a metà degli anni Novanta erano rare le ordinarie, poche e pochi associati molto motivati a livello personale e politico, le e i quali - tranne il caso meno svantaggiato delle sociologhe, delle angliste, di qualche storica - hanno dovuto confrontarsi non solo con la mentalità ostile e arretrata di interi dipartimenti e facoltà, ma con la macchina familistico-mafiosa dell'università italiana ${ }^{28}$. Soltanto

vocesociologica.it/tabella-categoria/5I-carriere\%2odonne> (data di ultima consultazione 8 dicembre 20I2) commentava che nella graduatoria del "gender gap index» l'Italia era all' $84^{\circ}$ posto su I2 8 paesi e ultima in Europa. Nella stessa classifica la Spagna si collocava al Io $^{\circ}$ posto.

28. È stato il libro-denuncia best seller dei giornalisti del «Corriere della sera» Sergio Rizzo e Gian Antonio Stella, La casta, Milano, Rizzoli, 2007 (I.200.00o copie vendute) a rendere di pubblico dominio una situazione ben nota a chiunque abbia lavorato anche per poco tempo nelle università italiane. Cfr. anche, degli stessi, $L a$ deriva, Milano, Rizzoli, 2008. Sulla crisi dell'università, esplosa con la riforma Berlinguer, cfr. Maurizio Ferraris, Una Ikea di università, Milano, Raffaello Cortina, 200I (nuova edizione 2009), e Roberto Perotti, L'università truccata, Torino, Einaudi, 2008. Cfr. inoltre la raccolta di articoli critici sull'università in "Donne e Ricerca», 9, I999, tra le rare pubblicazioni che abbiano affrontato i problemi della sofferenza delle donne all'interno delle istituzioni universitarie; una valutazione a distanza di dieci anni intorno all'atteggiamento imbarazzato e 
dalla fine degli anni Ottanta in poi si è avviata una faticosissima scalata, invariabilmente 'sotto mentite spoglie'. Per via della ottocentesca struttura dei programmi di insegnamento allora vigenti nell'università italiana — modificati soltanto dopo il 2000 - all'interno di quelli che un tempo erano chiamati piani di studio i nomi dei corsi insegnati erano il risultato di una procedura di ammissione approvata dalle facoltà e dal senato accademico, che allora non avrebbero mai acconsentito a introdurre un corso ufficiale intitolato alle donne. Si tratta di due decenni orsono, ma senza esagerazioni chi l'ha vissuto ricorda che il clima prevalente nell'establishment accademico non era poi molto diverso da quello che un secolo prima aveva animato l'antisuffragismo.

Una data precisa segna la svolta: il 1987. Nel marzo di quell'anno si svolse a Modena il primo appuntamento nazionale sugli studi delle donne in Italia. Vale la pena di ricordare, inoltre, che almeno per i successivi dieci anni, o giù di lì, è stato l'unico incontro a riunire insieme sia donne di provenienza e di formazione culturale e scientifica molto diversa (scienziate, letterate, antropologhe, storiche, filosofe, psicoanaliste, sociologhe ed economiste), che esponenti di centri sociali, sindacati, gruppi di attiviste; alla presenza di un pubblico sia interno che esterno al mondo accademico. In questo convegno per la prima volta femministe che lavoravano all'università hanno presentato a un affollato auditorio pieno di aspettative politiche, culturali e professionali il risultato di oltre quindici anni di ricerche, militanze, collaborazioni e confronti nella sfera pubblica e con le istituzioni ${ }^{29}$. Se il bilancio tracciato indicava un grande successo sul piano degli sforzi investiti e dei risultati di ricerca, quasi inesistenti da registrare erano le conquiste sul piano istituzionale: in tutta Italia c'era un solo corso affidato a una docente non inquadrata ufficialmente, la grande maggioranza delle relazioni presentate al convegno erano di ricercatrici intorno ai 40 anni, poche le associate intervenute, una sola ordinaria.

Considerata tra le scarse soluzioni disponibili per sopravvivere nel paese dei mandolini, la strategia del travestimento sembrò allora una delle poche strade percorribili, tenuto conto che trent'anni fa, come anche oggi, chi lo desidera può svolgere corsi e seminari anche sui temi in apparenza più scandalosi. Basta che lo faccia 'sotto mentite spoglie', vale a dire, mimetizzata/o dietro innocue etichette dall'aria perbene approvate

talvolta ostile con cui il fascicolo venne accolto si trova in Paola Di Cori, Franca Balsamo, Il libretto bianco. Postscriptum, in "Achab», I5, ottobre 2010 (www.achabrivista.it, data di ultima consultazione 8 dicembre 20I2).

29. Gli atti sono raccolti in un volume a cura di Maria Cristina Marcuzzo e Anna Rossi-Doria, La ricerca delle donne. Studi femministi in Italia, Torino, Rosenberg \& Sellier, 1987. 
dalle facoltà. Nella tradizione didattica predominante in Italia, in particolare nelle facoltà umanistiche e in quelle di scienze sociali, si utilizza lo strumento del syllabus non come mezzo con cui si disegna un piano di lavoro, bensì come indicazione generica del tema che sarà trattato. Scorrendo i programmi dei corsi insegnati nelle facoltà umanistiche delle università italiane ancora oggi si ha spesso la sensazione di leggere brevi liste della spesa prive di significato, dietro le quali si può trovare ciò che si vuole: una specie di elenco del telefono, organizzato con criteri medievali - nel senso delle classificazioni del sapere universale che compaiono, per esempio, nelle Etimologie del vir secolo del dottore della chiesa Isidoro di Siviglia; lunghi inventari di argomenti trattati da Tizia o Caio ${ }^{30}$.

Siccome la maggioranza delle femministe universitarie appartenevano ai livelli bassi della struttura accademica, fin dalla metà degli anni Settanta ha avuto inizio un periodo di doppio regime: da un lato si avviava una più decisa strategia di infiltrazione, dall'altro si rafforzavano i desideri (sacrosanti, ma non dichiarati nel paese della «dissimulazione onesta») di autopromozione accademica. Inoltre, a mano a mano che questi studi cominciavano anche ad avere qualche leggero riconoscimento sul piano pubblico e politico (diciamo dalla metà degli anni Ottanta in poi), alcune donne meglio inserite nella gerarchia accademica - con ambizioni di carriera e di affermazione, e con scarse possibilità di ottenerle attraverso le vie tradizionali — le quali fino ad allora avevano avuto solo occasionali rapporti con il femminismo, e ancor meno con i «women's studies» e poi «gender studies» del mondo anglofono, si sono candidate a rappresentare questi studi nel confronto con le istituzioni; con molti oneri (ma anche qualche onore) hanno svolto un indispensabile ruolo di addomesticamento, rendendoli accettabili a un consesso i cui pregiudizi bene riflettono quelli che caratterizzano l'attuale classe dirigente italiana.

Come mai ho voluto insistere sull'elemento 'lavorare sotto mentite spoglie'? Perché penso che questa difficoltà - dover nascondere, mascherare, occultare, imbellettare, rendere commestibile — ciò che ciascuna

30. Basta aprire le pagine web di qualsiasi università italiana per verificare questo dato. Negli Stati Uniti si possono al contrario trovare syllabus di diverse pagine che non danno soltanto una bibliografia esauriente, ma suddividono il carico di lavoro degli studenti lezione per lezione. Assai originale il syllabus preparato da David Foster Wallace per gli studenti del corso di letteratura da lui insegnato nel 1994 in una università dell'Illinois, che è stato pubblicato sul supplemento domenicale di «Repubblica» l'in dicembre 20II. Ma si vedano per esempio il bellissimo syllabus (undici pagine) dedicato a storia e memoria del professor Jay Winter per il semestre di primavera del 200 i presso la Columbia University: <www.columbia.edu/cu/history/pdf/.../g8325.pd> (data di ultima consultazione 8 dicembre 20I2) o quello assai bene articolato di Silvia Stoller dedicato a Luce Irigaray dell'autunno 2009 presso l'Università dell'Oregon: <http://philosophy.uoregon.edu/Syllabus_PHIL_463_563_ Irigaray.htm> (data di ultima consultazione 8 dicembre 20I2). 
ricerca e insegna, ha significato introdurre $\mathrm{i}$ «gender studies» a un costo personale e professionale altissimo, e in molti casi i risultati ottenuti a prezzo di sotterfugi e travestimenti sono stati inadeguati e frustranti rispetto alle energie investite. Ha costretto a deformare e mortificare le potenzialità di questi studi al fine di riuscire a insegnarli, inserirli nei programmi e affermarli. Il fatto di travestirli per renderli accettabili, truccarli al fine di renderli simili a quelli 'normali', è stato un elemento frenante: dato che non si riusciva a trovare un nome appropriato per loro nel piano di studi, era anche difficilissimo costruire alcune indispensabili alleanze interdisciplinari, promuovere seminari, programmi di ricerca e didattica con la libertà consentita per i corsi regolari. Più di altre ragioni, la strategia del mascheramento ha impedito di poter presentarsi al corpo studentesco, alle donne più giovani, alle colleghe, ai consigli di facoltà, con il volto scoperto e non velate. Insegnare studi di genere sotto copertura era come essere costrette a indossare un burqa immaginario.

Inoltre, la strada dell'incrocio tra saperi e pratiche di natura mista - essenziale per determinare una identità né disciplinare né disciplinata tipica degli studi di genere - fu resa di fatto poco praticabile. Se per introdurli era necessario nasconderli all'interno della propria specializzazione di provenienza, era difficile costruire programmi di didattica al di fuori di questa; la complicata strategia di elaborazione trasversale dei saperi relativi a generi e sessualità ne fu così profondamente compromessa. A sua volta, per ironia della sorte ciascuna finiva anche per perdere la fisionomia che aveva avuto un tempo, quella definita dalla appartenenza a una casella ministeriale specifica a fini concorsuali; in effetti, dopo vent'anni di dedizione a studi delle donne e di genere senza passaporto, erano stati anche strappati i vincoli con le proprie origini scientifiche neutre. Chi stava dentro le università non ha potuto fare un concorso (cercando anche di vincerlo) che a prezzo di travestimenti e compromessi anche degli argomenti studiati, ubbidendo alle regole vigenti in Italia che premiano ubbidienza e fedeltà, parentele, rete di amicizie influenti, alleanze di facoltà, come è stato prima ricordato. In questo modo coloro che si richiama(va)no a studi LGBTQ e nell'attualità rimangono ancora in posizioni marginali e deboli, sono state/i condizionate/i fortemente nei temi che studiavano, nelle maniere con cui orientavano il proprio percorso scientifico a livello metodologico e teorico, oltre che penalizzate/i sul piano professionale. La soluzione all'italiana, anziché costituire un esempio di pragmatismo, ha finito quindi per mettere in luce debolezze e passività di un intero sistema non facilmente estirpabili. 
Per finire, accenno solo di sfuggita a un aspetto importante che meriterebbe invece un'attenzione specifica: quello 'di classe', per usare un termine desueto che la crisi economica ha riportato in auge. Pur nati e sostenuti accademicamente e politicamente soprattutto dai ceti medio-bassi, gli studi di genere in Italia si sono affermati privilegiando alleanze sociali e familiari che hanno favorito le classi medio-alte. L'università italiana non è stata quasi mai un canale privilegiato di promozione sociale, in particolare per l'area umanistica; e per tutti i motivi che abbiamo illustrato precedentemente, se si legge la situazione con la lente del reddito e delle parentele influenti una ricerca sulla appartenenza sociale di chi ha insegnato studi di genere nel corso degli ultimi decenni mostrerebbe che le proverbiali difficoltà di affermazione delle italiane nell'università, anziché diminuire, sono cresciute; che irrobustire autonomia ed emancipazione rimangono per le donne obiettivi importanti.

\section{Quale domani?}

Detto tutto questo. Cosa fare? Come, dove guardare?

Il primo capitolo introduttivo di un bel libro di conversazioni tra Jacques Derrida e Elizabeth Roudinesco, Quale domani? ${ }^{31}$, tocca un punto che mi sta a cuore e che vorrei proporre come tema da dibattere in questo convegno. Si intitola: Scegliere la propria eredità.

Quando il colloquio tra i due ha luogo siamo nel 2000-200I. Il filosofo e la psicoanalista sono ormai maturi e dialogano su cosa fare nei confronti della grande tradizione filosofica e culturale che hanno alle spalle. Cosa fare di Althusser, di Foucault e di Barthes, di Lacan, Irigaray e Kristeva? E aggiungerei anche, perché li amo molto, di Sarah Kofman e di Michel de Certeau, e di altre/i attivi in Francia fino agli anni Ottanta? Roudinesco riassume all'inizio le posizioni di entrambi per osservare: «né accettare tutto, né fare tabula rasa» (p. II). A sua volta, prendendo lo spunto dalla coppia fedele/infedele implicita in questa frase, Derrida si sofferma a precisare le modalità di una operazione simile a quella che sta anche al centro del femminismo, di quello che è rimasto e anche di come viene vissuto oggi: essere infedeli per rimanere fedeli.

[...] è necessario - dice Derrida - fare tutto il possibile per appropriarci di un passato che sappiamo bene come resti in fondo inappropriabile - che si tratti di

3I. In francese l'interrogativo non c'è: De quoi demain... Dialogue. Cfr. Jacques Derrida, Elizabeth Roudinesco, Quale domani?, Torino, Bollati Boringhieri, 2004 (edizione originale 2003). 
memoria filosofica, della preminenza di una lingua o di un processo di filiazione in genere. "Riaffermare» - che cosa intendiamo con questo termine? Non soltanto accettare questa eredità, ma darle un nuovo impulso mantenendola in vita. Non sceglierla — perché ciò che caratterizza un'eredità è proprio il fatto di non poterla scegliere, mentre è essa che ci sceglie in modo del tutto arbitrario e, per così dire, violento - ma scegliere di mantenerla in vita. La vita, in fondo, il fatto di essere in vita, si definisce proprio in virtù di questa tensione interna che è tipica dell'eredità, attraverso una reinterpretazione di quel dato che è il dono e persino il processo di filiazione. Questo processo di riaffermazione che è al tempo stesso una prosecuzione e un'interruzione, è riconducibile, comunque, a una scelta, a una selezione, a una decisione. Propria come altrui: come due firme allo specchio, una di fronte all'altra. (p. I5)

Vorrei accostare a questa un'altra citazione che ho sempre tenuto presente fin da quando l'ho letta per la prima volta molti anni fa. Si tratta di un aforisma assai famoso di René Char che Hannah Arendt commenta a lungo nella introduzione alla raccolta Tra passato e futuro: "La nostra eredità non è preceduta da alcun testamento» («Notre héritage n’est précedé d'aucun testament»). Qui siamo nel I96I; Arendt ragiona sul significato che la tradizione aveva per coloro che avevano preso parte alla Resistenza: un vuoto; un vuoto di memoria di fronte a quella "apparizione di libertà» comune agli e alle resistenti. E accosta all'aforisma di Char una altrettanto nota parabola di Kafka, il frammento intitolato Egli:

Egli ha due avversari; il primo lo incalza alle spalle, dall'origine, il secondo gli taglia la strada davanti. Egli combatte con entrambi. Veramente, il primo lo soccorre nella lotta col secondo perché vuole spingerlo avanti, e altrettanto lo soccorre il secondo nella lotta col primo perché lo sospinge indietro. Questo però solo in teoria, perché non ci sono soltanto i due avversari ma anche lui stesso: e chi può dire di conoscere le sue intenzioni? Certo, sarebbe il suo sogno uscire una volta, in un momento non osservato - è vero che per questo ci vuole una notte buia come non è stata mai dalla linea di combattimento, e per la sua esperienza nella lotta essere nominato arbitro dei suoi avversari, che combattono tra loro. ${ }^{32}$

Sono parole che dicono moltissimo a noi oggi; non solo alla mia generazione, ma anche a chi è giovane. Commentando queste pagine di Arendt nella introduzione al libro, Alessandro Dal Lago scrive che «il tesoro della libertà dell'agire è impossibile da trasmettere in un mondo che non attribuisce senso all'agire in pubblico» (p. 18). Questa osservazione è preziosa se teniamo conto delle osservazioni fatte in precedenza. Senza combattere a viso scoperto, nell'arena pubblica, per il nostro tesoro, qual è il prezzo da pagare, quanto alto quello che abbiamo pagato?

32. Hannah Arendt, Tra passato e futuro, Milano, Garzanti, I999 (edizione originale 196I). 
Per fortuna, nonostante le considerazioni critiche svolte fin qui, gli studi di genere si sono diffusi, e nelle forme più diverse, come testimonia anche la varietà di temi e approcci esplicitata nel programma che abbiamo da svolgere nelle due giornate di convegno. Volendo dare qualche indicazione di massima e fare qualche passo in avanti, sarebbe probabilmente necessario adottare un insieme di strategie diversificate: rafforzare le situazioni e i contesti nei quali questi studi sono presenti solo in forme assai marginali da un lato; intervenire creativamente e criticamente laddove esistono in forme poco efficaci, appiattiti sul conformismo culturale e accademico dall'altro.

Un giovane amico che sta prendendo un dottorato a Leeds al quale ho mandato il programma del convegno - Francesco Ventrella - mi ha risposto con un commento che riesce a riassumere con acutezza alcune questioni centrali su cui occorrerà continuare a interrogarsi per molto tempo a venire:

[...] in Italia gay, lesbiche e trans sono diventati visibili ma l'omofobia dilaga; la politica e le istituzioni sono piene di donne, ma il sessismo è rampante; gli studi di genere e gli studi queer si insegnano da Palermo a Udine e la cultura italiana non è mai stata così sessista. Insomma, la cultura di genere dentro e fuori dalle università in Italia è una «concessione», avrebbe detto Carla Lonzi, del mito culturale. È perché il femminismo mascherato e pendolare non è più sostenibile di fronte al desiderio di studenti e studentesse di essere 'out'? - ma essere 'out' senza davvero criticare la costituzione estetica, narrativa, politica di questo 'outness' che senso ha? Vorrei tanto avere delle risposte in questo periodo, e invece ho solo domande. ${ }^{33}$

33. Messaggio spedito per e-mail il 20 novembre 20 Io. 